\title{
La reconnaissance administrative du handicap mental : entre respect de l'égalité et prise en compte de l'individu
}

Legal Recognition of People with Non-Physical Disabilities: Respect for equality and taking account of the individual

\section{Adèle Bourdelet}

\section{OpenEdition}

Journals

Édition électronique

URL : http://journals.openedition.org/add/789

DOI : $10.4000 /$ add. 789

ISSN : 2606-1988

\section{Éditeur}

Presses universitaires de Rouen et du Havre

\section{Édition imprimée}

Date de publication : 1 avril 2015

Pagination : $35-60$

ISSN : 1955-0855

Référence électronique

Adèle Bourdelet, «La reconnaissance administrative du handicap mental : entre respect de l'égalité et prise en compte de l'individu », Les Annales de droit [En ligne], 7 | 2013, mis en ligne le 03 mai 2018, consulté le 30 avril 2019. URL : http://journals.openedition.org/add/789 ; DOI : 10.4000/add.789 


\title{
La reconnaissance administrative du handicap mental : entre respect de l'égalité et prise en compte de l'individu
}

\author{
Adèle BOURDELET
}

The moral test of government is how that government treats those who are in the dawn of life, the children; those who are in the twilight of life, the elderly; those who are in the shadows of life; the sick, the needy and the handicapped ${ }^{1}$.

La Déclaration des droits de l'homme et du citoyen, partie intégrante du bloc de constitutionnalité français ${ }^{2}$, proclame que «les hommes naissent et demeurent libres et égaux en droits ». Dans l'esprit de penseurs libéraux comme Friedrich Hayek, cette égalité en droit s'oppose à la différenciation dans le traitement des citoyens par catégorie. Cette différenciation est fondée sur "l'égalité matérielle», incompatible avec l'égalité en droit dans la mesure où l'inégalité matérielle résulte justement de l'égalité en droit, les citoyens ayant fondamentalement des aptitudes différentes ${ }^{3}$.

Cependant, le Conseil d'État a pu parler d'une véritable «tradition française » de l'intérêt général volontariste. Celui-ci s’inscrit justement

1. «Ce qui éprouve la moralité d'un gouvernement est la manière dont ce gouvernement traite ceux qui sont à l'aube de la vie, les enfants; ceux qui sont au crépuscule de la vie, les personnes âgées; ceux qui sont dans l'ombre de la vie, les malades, les nécessiteux, les handicapés » (Hubert H. Humphrey, discours du $1^{\text {er }}$ novembre 1977 à l'occasion de l'inauguration du Hubert H. Humphrey building, Congressional Record, 4 novembre 1977, vol. 123, p. 37287.

2. Conseil constitutionnel, décision $n^{\circ} 73-51$ DC du 27 décembre 1973, Loi de finances pour 1974.

3. Friedrich Hayek, Droit, législation et liberté, Paris, PUF, «Quadrige, Grands Textes», 2007, p. 484. 
dans l'idée que l'intérêt général ne se limite pas à l'arbitrage entre des intérêts individuels, mais a la vocation plus large de garantir à chaque membre de la société des conditions d'existence dignes ainsi que l'accès à certains services considérés comme essentiels. Cela justifie donc un traitement catégoriel afin de rétablir, a minima, une égalité matérielle entre les membres de la société qui la composent ${ }^{4}$.

Pour Jacques Chevallier, « cette égalisation des conditions d'existence, l'État providence la poursuit en jouant sur trois registres» :

L'universalisme, veillant à ce que chacun soit garanti contre les risques de l'existence, l'État providence fournit à tous un ensemble de prestations en assurant des conditions égales d'accès (les "services publics»). La redistribution: des transferts sociaux sont opérés en faveur des plus défavorisés. Le particularisme: des politiques sociales sont construites en direction de «publics-cible» particulièrement vulnérables ${ }^{5}$.

Ce qui nous intéresse, dans un premier temps, c'est de comprendre les mécanismes qui amènent d'abord à définir une catégorie pour ensuite envisager d'engager une politique publique spécifique à cette catégorie. Nombreuses sont les politiques publiques dirigées vers une catégorie spécifique d'usagers ${ }^{6}$, dont beaucoup en raison d'une vulnérabilité supposée ou réelle: c'est notamment le cas pour les enfants, les femmes mais c'est également vrai pour les personnes handicapées. S'il est relativement aisé d'identifier ce qui constitue la catégorie des femmes, certaines autres catégories ont des contours beaucoup plus flous. C'est le cas du handicap. Ainsi, si, dans les représentations collectives, la personne paraplégique en fauteuil roulant est handicapée, à tel point que le fauteuil est devenu un symbole couramment utilisé, qu'en est-il en revanche des handicaps «invisibles »? Aussi il apparaît tout d'abord nécessaire de définir ce qu'est le handicap.

Il apparaît que la définition même du handicap porte en elle cette reconnaissance d'une vulnérabilité particulière. En effet, le terme de handicap vient de l'anglais hand in cap qui signifie littéralement « une

4. «Nul doute que la tradition française, telle qu'elle s'exprime dans la législation et la jurisprudence, a clairement pris le parti de promouvoir un intérêt général qui aille au-delà d'un simple arbitrage entre intérêts particuliers. Elle s'inscrit, sans conteste, dans la filiation volontariste de l'intérêt général » (rapport public du Conseil d'État, Réflexions sur l'intérêt général, 1999).

5. Jacques Chevallier, "L'État régulateur», dans Martine Lombard (dir.), Régulation économique et démocratie, Paris, Dalloz, "Thèmes et commentaires», 2006, p. 33.

6. Voir notamment Olivia Bui-Xan, dans Denys de Bechillon et Nicolas Molfessis (dir.), Le Droit public français: entre universalisme et différencialisme, Paris, Economica, «Corpus essais », 2004. 
main dans le chapeau » et était utilisé, originellement, dans les courses de chevaux pour faire référence à un désavantage supplémentaire supporté par certains concurrents. Aussi, le handicap apparaît non pas comme une cause mais une conséquence : le handicap est la conséquence sociale d'un désavantage particulier supporté par un individu. Aujourd'hui, la loi du 11 février $2005^{7}$ identifie cinq types de handicap : le handicap physique, le handicap sensoriel, le handicap cognitif, le handicap psychique et le handicap mental.

Le handicap mental peut alors se définir comme les conséquences sociales d'une déficience intellectuelle ${ }^{8}$. Or, l'identification de la déficience intellectuelle n'est pas sans poser de problèmes, ce qui explique, en partie la tardiveté de la reconnaissance du handicap mental. Dès le début du XviII ${ }^{\mathrm{e}}$ siècle, notamment avec Jean Itard ${ }^{9}$, se développe l'idée de l'éducabilité des enfants atteints d' $\mathrm{d}^{\text {idiotisme }}{ }^{10}{ }$. Si cela constitue une première avancée dans l'identification de la déficience intellectuelle ainsi que dans l'idée de la nécessité d'une prise en charge spécifique, le problème reste encore largement un problème privé, tout comme d'ailleurs, l'éducation en général ${ }^{11}$. Il faudra en effet attendre le développement non seulement des sciences cognitives à la fin du XIX ${ }^{\mathrm{e}}$ siècle, mais également le développement de l'État providence au début $\mathrm{du} \mathrm{xx}^{\mathrm{e}}$ siècle, pour que puisse être progressivement identifiée la déficience intellectuelle et que le handicap mental puisse, partant de là, être lui-même reconnu. La première échelle métrique de l'intelligence ${ }^{12}$ n'a ainsi été créée qu'en 1905. Parallèlement, sous l'influence, notamment, des découvertes de Charles Darwin sur la sélection naturelle, concomitantes à celles de Gregor Mendel sur les lois de l'hérédité, se développe l'idée de l'eugénisme, qui mènera aux dérives que l'on connaît lors de la seconde guerre mondiale. Le rejet des actes commis pendant cette période, notamment à l'égard

7. Loi $n^{0}$ 2005-102 du 11 février 2005 pour l'égalité des droits et des chances, la participation et la citoyenneté des personnes handicapées.

8. Les maladies mentales entrant d'avantage dans le champ du handicap psychique, et des troubles comme l'autisme ou la dyslexie appartenant quant à eux au champ du handicap cognitif.

9. Jean Itard est le médecin qui en 1801 prit le pari de l'éducabilité de Victor, l'enfant sauvage de l'Aveyron. Voir notamment Lucien Malson, Les Enfants sauvages : mythe et réalité, Paris, 10/18, 2002.

10. Terme alors utilisé pour désigner la déficience intellectuelle.

11. La loi rendant l'instruction obligatoire ne datant en effet que de 1882 (loi $\mathrm{n}^{\circ}$ 11-696 du 28 mars 1882 qui rend l'instruction primaire obligatoire).

12. Encore aujourd'hui utilisée, bien que modifiée, sous le nom d'échelle de BinetSimon et constituant l'un des instruments en matière de tests psychométriques, appelés communément tests de quotient intellectuel. 
des personnes handicapées mentales et psychiques placées en asile, va favoriser l'émergence d'associations luttant pour la reconnaissance des droits individuels des personnes handicapées mentales. Ces nouveaux acteurs entrent en jeu à partir des années 1950. Il s'agit principalement d' associations de parents comme l'Union nationale des parents d'enfants inadaptés ${ }^{13}$ (UNAPEI) ou Papillons blancs, qui luttent pour la reconnaissance des droits individuels des personnes handicapées mentales notamment un droit au travail. C'est ainsi qu'elles vont créer le premier centre d'aide par le travail (CAT) en 1954.

La reconnaissance progressive de la déficience intellectuelle, du fait à la fois des avancées scientifiques et des acteurs privés, va amener aux premières lois sur la prise en charge du handicap. Cependant, il devient alors nécessaire pour l'administration de cibler ceux qu'elle entend reconnaître comme handicapés. La réponse apportée est alors la création d'institutions chargées de reconnaître la qualité de personnes handicapées et c'est de cette reconnaissance que dépendra l'attribution d'aides spécifiques à destination des personnes handicapées.

La première loi mettant en place une institution capable de reconnaître à une personne la qualité de personne handicapée et notamment celle de travailleur handicapé est la loi du 23 novembre $1957^{14}$, qui crée les commissions départementales d'orientation des infirmes (CDOI) chargées de reconnaitre le handicap et d'orienter en conséquence la personne handicapée. Dès l'origine, l'institution chargée de cette reconnaissance administrative est mise en place au niveau local, plus précisément au niveau des départements. Dans le cadre d'un État très fortement centralisé, il s'agit naturellement d'administrations déconcentrées, directement sous l'autorité des ministères centraux intéressés. Ce sera encore le cas avec la loi du 30 juin 1975 en faveur des personnes handicapées, créant les commissions techniques de reclassement professionnel (COTOREP) chargées de reconnaître le handicap des majeurs, ainsi que les commissions départementales de l'éducation spéciale (CDES), spécialisées dans la reconnaissance du handicap chez l'enfant. Chacune de ces commissions ayant comme rôle principal de reconnaître la qualité de personne handicapée à un usager et de fixer un taux d'incapacité

13. Si le sigle originel a été conservé par l'UNAPEI, sa signification est aujourd'hui différente. Il s'agit désormais de l'union nationale des associations de parents et amis de personnes handicapées mentales.

14. Loi $\mathrm{n}^{\mathrm{O}}$ 57-1223 du 23 novembre 1957 sur le reclassement professionnel des travailleurs handicapés. 
correspondant, ainsi que de déterminer l'orientation de cette personne selon la gravité et la nature de son handicap.

Or la loi du 11 février $2005^{15}$ est venue profondément bouleverser les institutions existantes ainsi que la procédure de reconnaissance du handicap par l'administration. Notamment les CDES et COTOREP ont disparu au profit d'une institution unique : les maisons départementales des personnes handicapées (MDPH). Il ne s'agit pas là d'une simple fusion, mais d'une véritable réorganisation qui apparait comme une illustration d'un enjeu de plus en plus prégnant dans l'administration: la recherche de l'efficacité de l'action administrative.

Dès 1982, Paul Amselek, dépeignait les grands traits de l'évolution de la technique juridique dans les sociétés occidentales par deux principales caractéristiques : la tendance d'une part à la direction souple et autonome de la conduite humaine par opposition à une conduite autoritaire et hétéronome ${ }^{16}$. En d'autres termes, les instruments juridiques prenaient de plus en plus la forme de recommandations non contraignantes, laissant une marge de manœuvre aux gouvernés ${ }^{17}$ et ils associaient les gouvernés eux-mêmes, voire en émanaient. On peut avancer que ces deux tendances de l'évolution des instruments juridiques qui caractérisent la direction des conduites humaines se retrouvent désormais au cœur des nouveaux modes de l'action administrative. "Associer ceux que la décision concerne, et qui devront la traduire dans les faits [...] » : telle est, selon Jean Rivero, «la méthode qui paraît la mieux adaptée à l'efficacité de l'action ${ }^{18}$ ». Seulement, il est d'abord nécessaire d'identifier « ceux que la décision concerne». Il s'agit bien évidemment des personnes handicapées elles-mêmes, mais aujourd'hui, avec le processus de décentralisation et le transfert d'une part importante des compétences sociales aux départements, il s'agit également des collectivités territoriales qui financent une partie des aides, ainsi que de nombreux établissements relatifs à la prise en charge des personnes handicapées mentales. La nouvelle institution qu'est la MDPH poursuit justement cet objectif d'associer à la décision l'ensemble des acteurs, publics ou privés, concernés

15. Loi $\mathrm{n}^{0}$ 2005-102 du 11 février 2005 pour l'égalité des droits et des chances, la participation et la citoyenneté des personnes handicapées.

16. Paul Amselek, "L'évolution générale de la technique juridique dans les sociétés occidentales », Revue de droit public, 1982-1, p. 285-294.

17. Paul Amselek prenait l'exemple du domaine économique, où ce mode de direction juridique permet de concilier le rôle de l'État providence avec l'exigence de la liberté d'entreprise.

18. Jean Rivero, "Action économique de l'État et évolution administrative", Revue économique, vol. 13, n ${ }^{0}$ 6, 1962, p. 886-896. 
par l'application de la politique publique du handicap. Cependant cette association fait apparaître un risque pour la cohérence nationale en matière de politique publique du handicap. En effet, l'association de nouveaux acteurs au processus de décision, qu'il s'agisse des départements, des usagers ou même d'autres acteurs privés, tend à mettre en évidence des risques de conflit d'intérêt: l'intérêt individuel est potentiellement différent de l'intérêt général, et l'intérêt général peut apparaitre différent de l'intérêt public local. Or la réglementation paraît insuffisante, à elle seule, à contenir ce risque, dans la mesure où cette réglementation laisse volontairement une marge de manœuvre aux MDPH afin d'évaluer au cas par cas la situation des demandeurs. Cela explique que, dans un tel contexte de diversification des acteurs de la décision, les mécanismes de régulation, entendus comme encadrement non contraignant de la décision, prennent une part de plus en plus importante, mais cependant complémentaire à la réglementation, ce qui peut apparaître comme le deuxième trait caractéristique de l'évolution de l'action administrative, y compris concernant l'action spécifique à destination des personnes handicapées ${ }^{19}$.

\section{L'intégration de nouveaux acteurs à la procédure de décision de reconnaissance administrative du handicap mental : I'autonomisation de la politique publique du handicap vis-à-vis du pouvoir étatique}

Les MDPH sont désormais, selon les termes du Code de l'action sociale $^{20}$, des groupements d'intérêts publics. Le choix d'une telle forme d'organisation pour une institution publique permet potentiellement de faire intervenir une infinité d'acteurs dans la phase préalable à la décision, notamment des acteurs privés. Concernant la décision elle-même, la composition de l'organe de décision des MDPH, la Commission des droits et de l'autonomie des personnes handicapées $(\mathrm{CDAPH})$, traduit également une diversification des acteurs à travers l'intégration des collectivités locales et des représentants d'usagers. Cette diversification des acteurs dans la reconnaissance du handicap tend à accréditer la thèse d'une autonomisation de la direction de la politique du handicap, au sens où l'entend Paul Amselek. Ainsi, cette direction ne dépendrait plus de la seule volonté de l'État, mais également de

19. Voir notamment le rapport public du Conseil d'État de 2001 sur les autorités administratives indépendantes.

20. Art. L 146-4 du Code de l'action sociale et des familles (CASF). 
celle de l'ensemble des personnes publiques ou privées concernées par l'application de cette politique, à commencer par les usagers eux-mêmes.

\subsection{Une infinité d'acteurs pouvant potentiellement intervenir dans la procédure préalable à la décision}

Depuis la loi du 11 février 2005, les COTOREP et CDES ont disparu au profit des $\mathrm{CDAPH}$, qui rassemblent désormais les attributions de ces deux instances. Ces commissions sont incluses dans un ensemble plus large appelé $\mathrm{MDPH}$, présentes dans chaque département. Ces MDPH comprennent, outre les commissions, un accueil, chargé d'orienter la personne, ainsi qu'une équipe pluridisciplinaire, chargée d'instruire le dossier de la personne handicapée. La forme adoptée par les MDPH est significative du choix opéré dans la conduite de la politique du handicap, dans la mesure où elle permet désormais l'intervention de l'ensemble des acteurs intéressés.

Les MDPH ont en effet été crées sous la forme de groupements d'intérêt public (GIP) sous la tutelle administrative et financière des départements ${ }^{21}$. Si elles n'ont pas la même autonomie que les GIP classiques du fait de cette tutelle, elles ont comme point commun avec l'ensemble des GIP qu'elles sont la mise en commun de moyens par différentes personnes publiques ou privées pour la réalisation d'une mission d'intérêt général à but non-lucratif. Contrairement aux anciennes CDES et COTOREP, qui étaient entièrement financées, pour leurs dépenses de fonctionnement, par l'État ${ }^{22}$, les MDPH ont la volonté d'associer l'ensemble des acteurs du handicap tant à la procédure de décision qu'au financement des moyens à mettre œuvre. C'est d'ailleurs l'objectif qui ressort de la circulaire ministérielle du 24 juin $2005^{23}$.

[Le] choix du statut de GIP a répondu à la nécessité de permettre une mise en synergie des moyens actuellement dédiés par l'État, les organismes locaux de sécurité sociale et les départements au dispositif public d'évaluation et d'orientation des personnes handicapées. Il s'agit aussi d'associer activement d'autres personnes morales, notamment des partenaires associatifs ou financiers, à la réalisation des missions et à la gestion de la maison départementale $e^{24}$.

21. Ibid.

22. Ancien article L 241-3 du CASF.

23. Circulaire DAGEMO-DAGPB-DGAS-DESCO du 24 juin 2005 relative au concours apporté par l'État au fonctionnement des maisons départementales des personnes handicapées.

24. Ibid. 
Les groupements d'intérêt public se caractérisent par la souplesse de leur gestion. S'il existe dans le Code de l'action sociale et des familles des règles de gestion communes pour l'ensemble des $\mathrm{MDPH}$, notamment quant à la composition, on retrouve cependant une certaine souplesse de gestion qui n'existait pas dans les anciennes CDES et COTOREP. Ainsi, la Caisse nationale des solidarités et de l'autonomie (CNSA) souligne que «la diversité des modalités d'organisation des MDPH est confirmée, d'une part dans les relations financières qu'elles entretiennent avec leurs partenaires, notamment lorsqu'on observe la part des dépenses propres dans le total des dépenses, et d'autre part, dans le nombre et l'origine des personnels qui y travaillent ${ }^{25}$ ». Le personnel des MDPH est en effet composé à la fois de fonctionnaires en détachement des trois différentes fonctions publiques et de contractuels de droit public recrutés par la $\mathrm{MDPH}^{26}$, ceci dans des proportions variables dans les différentes $\mathrm{MDPH}^{27}$. De même, cette souplesse de gestion se retrouve dans les financements des MDPH et les différents «partenaires» des $\mathrm{MDPH}^{28}$ qui les financent dans des proportions également différentes selon les départements. Ainsi les MDPH apparaissent comme des organes ayant d'emblée une autonomie les unes par rapport aux autres et vis-à-vis d'un pouvoir central hiérarchique. Ce pouvoir hiérarchique, à même d'imposer une unité et de supprimer les disparités, n'existe pas. À l'échelle nationale, il subsiste simplement un rôle de coordination concernant les règles d'organisation, dévolu principalement à la $\mathrm{CNSA}^{29}$, organe lui-même autonome vis-à-vis du pouvoir central. En définitive, si certaines personnes publiques, notamment l'État et les départements, sont obligatoirement associées au fonctionnement des MDPH, les partenaires associatifs et financiers auxquels fait référence la circulaire de 2005 précitée, peuvent également l'être. Or il n’est pas précisé de conditions particulières pour l'établissement d'une convention de partenariat entre une MDPH et l'un de ces partenaires. Le danger majeur qui apparaît alors est celui du lobbying, puisque rien n'empêche, en tout état de cause, l'établissement d'une convention avec un partenaire poursuivant un intérêt étranger à la fois à l'intérêt général et à l'intérêt catégoriel

25. CNSA, MDPH: 5 ans déjà! Synthèse des rapports 2010 des maisons départementales des personnes handicapées, dossier technique, décembre 2011.

26. Depuis la loi du 28 juillet 2011, dite « loi Blanc», ces contractuels peuvent désormais être recrutés en CDD comme en CDI.

27. CNSA, rapport précité.

28. Principalement la caisse nationale d'assurance maladie et les départements, mais également parfois l'AGEFIPH, la CNSA, voire dans certains cas les régions.

29. Voir infra. 
des personnes handicapées ${ }^{30}$. En effet, la difficulté particulière de la reconnaissance du handicap évoquée précédemment reste la délimitation de son champ. Les individus composant la société étant tous différents et dotés de talents différents peuvent dans leur totalité se prévaloir de difficultés relatives à ce qui fait leur spécificité. Ainsi une personne au physique disgracieux, sans être pour autant difforme ${ }^{31}$, peut se prévaloir d'une difficulté dans l'accès à l'emploi. Or l'intérêt des employeurs, par exemple, pourrait être, par une action d'influence, d'élargir la notion de handicap. Notamment celle de travailleur handicapé, qui leur permet de remplir leur obligation d'emploi sans pour autant avoir à employer des personnes lourdement handicapées. Cependant, un élargissement trop important de la notion de handicap tendrait à la vider de son sens, voire à la rendre inutile, et en définitive à rendre inutile l'action spécifique à destination des personnes handicapées, y compris celles qui le sont le plus lourdement. Si le lobbying peut présenter un danger, la possibilité offerte aux acteurs financiers de participer aux MDPH peut également avoir un intérêt. Ainsi il n'est pas exclu qu'un groupe financier propose gracieusement ses services ou mette à disposition des moyens afin de valoriser son image auprès de sa clientèle ou créer une cohésion au sein de l'entreprise autour de valeurs ${ }^{32}$ en mettant en avant son action en faveur des personnes handicapées, soulageant ainsi le coût de fonctionnement des MDPH et donc les dépenses publiques.

Si la participation d'une grande diversité d'acteurs est désormais rendue possible grâce à la forme de groupement d'intérêt public retenue pour les nouvelles MDPH, cette participation de nouveaux acteurs va au-delà de la simple mise en commun de moyens. En effet, si, originellement, seul l'État conservait le pouvoir décisionnaire concernant la reconnaissance de la qualité de personne handicapée, l'évolution de cette reconnaissance tend à donner à de nouveaux acteurs un poids de plus en plus lourd dans ce pouvoir de décision.

30. Le rôle néfaste du lobbying a notamment était souligné par le Sénat dans son rapport d'information sur l'amiante, rapport $\mathrm{n}^{\circ} 37$, annexe au procès-verbal de la séance du 30 octobre 2005.

31. La défiguration d'un individu, à la suite, par exemple, d'un incendie, étant en effet reconnue comme une cause de handicap par l'annexe 2-4 du CASF au titre de «handicap esthétique».

32. Voir notamment Anne Salmon, "Éthique et intérêt: quels mobiles pour "l'entreprise providence" ? ", Revue française de socio-économie, 2009-2, $\mathrm{n}^{\circ}$ 4, p. 39-57. 


\subsection{De nouveaux acteurs décisionnaires}

Si l'on a vu qu'une multitude d'acteurs pouvait s'associer aux MDPH et influencer les décisions, notamment au stade de l'instruction des dossiers, une autre question primordiale, qui permet d'évaluer la place de l'État et donc éventuellement de conclure à une direction autonome de la politique du handicap, est celle de savoir à qui appartient la décision finale de reconnaissance du handicap.

La MDPH n'est pas seulement une structure au sein de laquelle se prennent les décisions concernant la reconnaissance du handicap et l'orientation des personnes handicapées. Comme l'énonce les motifs de la loi de 2005, son objectif est plus large: les MDPH constituent «le guichet unique " auprès desquelles les personnes handicapées peuvent adresser l'ensemble de leurs demandes concernant leur handicap. Aussi est-ce un organe inclus dans la MDPH qui constitue l'organe décisionnaire : la CDAPH.

Pour parler d'évolution, il est tout d'abord nécessaire d'établir quelles étaient les modalités antérieures de décision, soit sous l'empire des anciennes CDES et COTOREP. Les deux organes ayant subi une évolution similaire en termes de représentation des usagers et des départements, nous nous pencherons plus spécifiquement sur le cas des COTOREP. Depuis leur création par la loi du 30 juin $1975^{33}$, les COTOREP ont toujours intégré en leur sein un représentant des départements, seul membre qui n'était d'ailleurs pas désigné par les préfets ${ }^{34}$. Seulement il s'agissait, jusqu'à un décret de $1995^{35}$, d'un représentant unique, noyé au milieu de dix-neuf autres représentants, choisis par le préfet et représentant en grande part les administrations déconcentrées. Le décret du 6 mai 1995 marque une première évolution puisqu'il porte ce nombre à trois. Ce nombre sera finalement porté à quatre avec la réforme de 2005. Plus spectaculaire est en revanche l'évolution de la représentation des usagers. En effet, jusqu'à la réforme de 2005, ces représentants n'étaient présents qu'au nombre de deux pour exploser brutalement à sept représentants avec la réforme de 2005.

Aujourd'hui la CDAPH comprend principalement à la fois des représentants de l'État et notamment de ses services déconcentrés, des représentants des départements ainsi que des représentants des usagers

33. Loi $\mathrm{n}^{\mathrm{O}}$ 75-534 du 30 juin 1975 d'orientation en faveur des personnes handicapées.

34. Ancien article D 323-3-1 du Code du travail.

35. Décret $n^{\circ} 95-642$ du 6 mai 1995 portant modification du code du travail ( $3^{\mathrm{e}}$ partie : décrets) relatif à la composition et au mode de fonctionnement des commissions techniques d'orientation et de reclassement professionnel. 
qui peuvent être considérés comme les acteurs principaux de la politique du handicap. Les évolutions successives, qui avaient déjà été entamées sous l'empire des COTOREP traduisent un recul du pouvoir central. Ainsi la CDAPH est composée de 23 membres, dont 4 représentants de l'État ${ }^{36}$ et de l'agence régionale de santé ${ }^{37}$ (ARS), 4 représentants du département et 7 représentants d'associations de personnes handicapées. Les 8 autres membres sont les représentants des caisses d'assurance maladie, d'organisations syndicales, des organisations gestionnaires d'établissements pour personnes handicapées, ainsi qu'enfin un représentant de parents d'élèves et un membre du conseil départemental consultatif des personnes handicapées. Enfin, au terme de l'article L 241-5 du CASF, les décisions peuvent être prises en formations restreintes. Au terme de l'article R 421-28 du CASF, ces formations restreintes sont composées d'au moins trois membres de la Commission ayant voix délibérative dont un représentant du département de l'État et un représentant du département. Étant donné la présence obligatoire d'un tiers de représentants d'usagers, il en résulte qu'au sein de ces formations restreintes sont toujours au moins représentés l'État, les départements et les usagers dans des proportions identiques, ce qui permet d'en déduire que ce sont désormais ces trois entités qui sont au cœur du dispositif de décision des CDAPH.

Si l'État reste toujours très présent, l'évolution entreprise montre un recul de sa présence puisque ces trois représentations (usagers, département, État) sont désormais présentes dans des proportions identiques, ce qui s'oppose à la prépondérance de l'État lors de la fondation des COTOREP. Un tel constat tend donc à démontrer l'autonomisation de la reconnaissance du handicap vis-à-vis du pouvoir étatique. La participation des départements aux MDPH apparaissait, d'un certain point de vue nécessaire. Car si le handicap a bien entendu une dimension médicale qui justifie l'intéressement de l'État, qui finance cet aspect à travers la Caisse d'assurance maladie, la dimension sociale du handicap, met quant à elle en jeu les compétences du département à travers l'aide

36. Issus de différents services déconcentrés de l'État : le directeur départemental chargé de la cohésion sociale, le directeur régional des entreprises, de la concurrence, de la consommation, du travail et de l'emploi, et enfin le directeur académique des services de l'Éducation nationale.

37. Depuis la loi $\mathrm{n}^{\mathrm{o}} 2011-901 \mathrm{du} 28$ juillet 2011 tendant à améliorer le fonctionnement des MDPH et portant diverses dispositions relatives à la politique du handicap. 
sociale ${ }^{38}$, compétence transférée depuis la loi Defferre du 7 janvier 1983. À ce titre, l'évolution de la représentation départementale depuis 1975 a suivi le processus de décentralisation et en est donc une résultante. Cette intégration est alors apparue comme d'autant plus nécessaire que l'État et les départements peuvent désormais avoir des intérêts divergents en matière d'attribution de certaines aides et orientations. Ainsi, par exemple, alors que les établissements et services d'aide par le travail ${ }^{39}$ (ESAT) sont des structures financées par l'État, les foyers occupationnels sont des structures financées par le département. Par conséquent, pour des causes strictement pécuniaires, il n'est pas impossible que l'État favorise l'orientation en foyer occupationnel, qui n'engage pas ses ressources, tandis que les départements favoriseraient l'orientation en ESAT. De même, les associations d'usagers sont elles aussi bien souvent gestionnaires d'établissements de type ESAT ou foyer occupationnel ${ }^{40}$ et pourraient tout à fait favoriser ou écarter l'admission éventuelle d'une personne handicapée dans l'un de ses établissements selon que la personne corresponde ou non à son projet d'établissement. De même, nous avons vu qu'il n'est pas toujours aisé, en raison de l'incertitude des contours du handicap mental, de discerner les personnes qui relèvent du handicap et donc de l'AAH et ceux relevant plutôt du dispositif de droit commun du minima social, le revenu de solidarité active (RSA). Or le RSA est un dispositif essentiellement financé par le département, tandis que l'AAH est essentiellement financée par l'État. Ce peut donc être une autre source de conflits d'intérêts. Quant aux associations d'usagers, elles auront une tendance naturelle à rechercher la meilleure intégration possible des personnes handicapées ainsi que le plus haut niveau de prestation.

La répartition équitable des voix délibératives entre État et département ainsi qu'une représentation des usagers à hauteur d'un tiers empêche que des décisions puissent être motivées uniquement par des

38. Ainsi, concernant le handicap mental, coexistent des structures à « double tarification » (maisons d'accueil spécialisées et foyers d'accueil médicalisés), ainsi que des structures exclusivement financées par le département (foyers occupationnels, dits aussi foyers de vie) selon le degré de médicalisation de la structure.

39. Nouvelle appellation des CAT depuis la loi de 2005.

40. Le CASF prévoit en effet que les établissements médico-sociaux puissent être gérés par des personnes morales de droit privé (art. L 312-1), sous réserve de l'agrément du directeur de l'ARS (art. L 313-3). Historiquement, nous avons vu que les premiers CAT avaient été crée par ce type d'association ce qui explique qu'elles soient encore très présentes en tant que gestionnaires de ces structures. 
questions budgétaires de l'un ou l'autre des principaux financeurs publics de la politique du handicap. Néanmoins, la place laissée à l'expression et à la conciliation entre des intérêts potentiellement divergents ne fait pas disparaître le risque pour l'unité des décisions. En effet, il faut tout d'abord souligner que les populations ne sont pas les mêmes d'un département à l'autre et n'ont donc pas les mêmes besoins, notamment en matière de prestations sociales ${ }^{41}$. Or, ces différences de population ont notamment comme effet que les départements ne mènent pas tous des politiques identiques en matière d'insertion sociale en général et de handicap en particulier, puisque les représentants de ces collectivités sont issus de la démocratie locale. Le principe de libre administration des collectivités territoriales, inscrit dans la Constitution depuis la réforme de 2003 a comme conséquence l'autonomie des départements non seulement vis-à-vis du pouvoir central ou des autorités déconcentrées, mais encore vis-à-vis des autres départements. Au sein des CDAPH peuvent donc librement s'exprimer des particularismes locaux ainsi que des actions de lobbying de la part des associations d'usagers. Ces éléments ne contribuent donc pas à l'égalité de traitement des usagers sur l'ensemble du territoire national. Cependant, dans le cas particulier de la reconnaissance du handicap, notamment mental, il ne s'agit pas de l'élaboration de dispositions réglementaires, mais de la prise de décisions administratives individuelles. Ainsi, si l'on peut considérer que l'opinion des départements comme celle des usagers devraient être pris en compte et peser dans l'élaboration des dispositions réglementaires à caractère général concernant le domaine du handicap, il peut apparaître plus contestable qu'ils soient associés à la prise d'une décision individuelle et encore plus qu'ils ne soient pas simplement associés mais décisionnaires, comme c'est ici le cas. Ainsi, dans le domaine réglementaire, concernant les dispositions relatives au fonctionnement des MDPH, si le gouvernement peut consulter différents acteurs de la politique publique concernée, la décision finale lui appartient.

En effet, nous l'avons vu, les usagers ont désormais le droit, et cela était d'ailleurs le cas sous l'empire des anciennes CDES et COTOREP, d'être entendus et donc associés à la procédure de décision. De plus, la généralisation de l'instauration d'un conciliateur ${ }^{42}$, permet désormais à

41. Voir notamment le rapport de la direction de la recherche, des études, de l'évaluation et des statistiques (DREES), «Les disparités départementales en matière d'aide sociale ", Études et résultats, $\mathrm{n}^{\circ} 269$, octobre 2003.

42. Dont l'instauration est d'ailleurs devenue, avec la loi de 2005, une obligation législative pour les MDPH. 
l'usager, par le biais d'une procédure gracieuse, de mieux comprendre la décision prise à son encontre, voire d'obtenir sa révision. Cependant, le fait de ne pas associer simplement des représentants d'usagers, ainsi que les collectivités locales, mais de leur donner véritablement un pouvoir décisionnaire relève d'une logique qui semble différente. C'est finalement faire de cette décision une décision négociée, négociation auquel le principal intéressé qu'est l'usager ${ }^{43}$, ne serait pas partie. Il s'agit d'une négociation entre l'État, représentant de l'intérêt général, le département, représentant d'un intérêt public local et les représentants des usagers, représentants d'un intérêt catégoriel. Ainsi la décision finale de la CDAPH n'est d'une certaine manière plus prise dans le seul intérêt général, mais dans un arbitrage d'intérêts différents, et donc potentiellement divergents. Pourtant le handicap devrait a priori être reconnu sur l'appréciation de causes objectives énoncées par les textes règlementaires ${ }^{44}$. Donner une place aux acteurs autres que l'État, non plus seulement au stade d'un dialogue préalable à la décision, mais également dans le cadre de la décision proprement dite, c'est également un constat de la part de subjectivité dans la reconnaissance du handicap. Cependant, il existe certaines limites au pouvoir décisionnaire de la $\mathrm{CDAPH}$ visant à atténuer les disparités entre les départements. Ces limites sont traditionnellement règlementaires et juridictionnelles mais passent aussi et désormais par une coordination nationale qui empêche un fonctionnement « en vase clos» de chaque MDPH prise isolément.

2. L'encadrement de la décision de reconnaissance administrative du handicap mental : un arbitrage entre la volonté d'individualisation de la reconnaissance du handicap et le respect de l'égalité de traitement des usagers

La volonté d'individualisation de la décision de reconnaissance du handicap, les incertitudes quant au champ de la reconnaissance du

43. L'usager lui-même pouvant avoir des intérêts divergents des représentants d'usagers, puisqu'il est porteur d'un intérêt individuel. D'autre part, concernant spécifiquement le handicap mental, il n'est pas à exclure que les représentants des usagers, qui sont en réalité d'avantage des représentants des familles d'usagers, poursuivent un intérêt différent des usagers eux-mêmes. Ainsi, l'inquiétude des parents pourrait les pousser à faire intégrer leur enfant dans une structure la plus sécurisante possible (comme un ESAT), alors même que le désir et les possibilités de l'usager lui permettraient une intégration en milieu ordinaire de travail.

44. Voir notamment, infra, le guide-barème. 
handicap, en particulier mental et enfin l'association de nouveaux acteurs, tant dans la procédure que dans la décision elle-même de reconnaissance du handicap, nécessitent un encadrement réglementaire doté d'une certaine souplesse. Cependant cette souplesse risque d'accentuer les disparités entre les décisions rendues par les différentes $\mathrm{CDAPH}$ ainsi que dans le fonctionnement même des MDPH. La réglementation apparaît donc insuffisante pour satisfaire à une cohérence nationale de la procédure de reconnaissance du handicap. Il est alors nécessaire de trouver de nouveaux modes d'actions non contraignants et incitatifs visant à coordonner l'action des acteurs de plus en plus nombreux dans le domaine du handicap, afin d'harmoniser les pratiques au niveau des départements ainsi que de garantir l'effectivité des décisions prises par les CDAPH.

\subsection{Un encadrement réglementaire souple}

L'irruption de la régulation dans la sphère des affaires sociales ne sous-entend pas nécessairement une déréglementation. En effet, si en matière d'économie la régulation est, dans les thèses libérales, associée à une certaine idée de déréglementation, c'est que le marché, censé engendrer une concurrence libre et non faussée, est perçu comme un meilleur régulateur que l'État lui-même. Or, en matière de politique sociale, l'État intervient cette fois dans une activité essentiellement non concurrentielle visant justement à soutenir des populations qui sont écartées du jeu du marché en raison d'une vulnérabilité particulière. Ce serait donc un non-sens que de prétendre que la concurrence apparaisse comme un régulateur des politiques sociales, puisque cette intervention de l'État résulte justement d'un constat de l'insuffisance de la libre concurrence à garantir un niveau de vie suffisant à l'ensemble de la société. La régulation est donc entendue ici d'un moyen nouveau propre à maintenir l'équilibre social ${ }^{45}$. Or, comme le constate Patrice Duran, "l'idée d'un pilotage centralisé a vécu quand le droit a perdu son côté systématique et la bureaucratie son autorité et sa compétence ${ }^{46}$ ». La régulation s'entend donc des procédés visant à maintenir un équilibre dans ce contexte d'une crise de l'autorité étatique. Cela se traduit

45. Jacques Commaille, «Régulation sociale», dans André-Jean Arnaud et al. (dir.), Dictionnaire encyclopédique de théorie et de sociologie du droit, Paris, LGDJ, 1993, p. 523 .

46. Stephan Breuer, «Max Weber et les formes de la démocratie», Revue européenne des sciences sociales, $\mathrm{n}^{0}$ 101, 1995, cité par Patrice Duran, «Légitimité, droit et action publique », L'Année sociologique, vol. 59, $\mathrm{n}^{\circ}$ 2, 2009, p. 303-344. 
donc par une association des différents acteurs directement concernés par la mise en œuvre de la politique en cause, mais également par l'assouplissement, et non par la disparition, du cadre réglementaire. Et cela afin justement de laisser une marge de manœuvre à ces différents acteurs.

La réglementation peut donc avoir pour objet la régulation, soit encadrer une direction souple d'une politique déterminée. Ainsi la décision de reconnaissance du handicap mental est fortement encadrée quant à la forme de la décision (composition de la MDPH, de la CDAPH...), mais l'est en revanche beaucoup moins sur le fond. C'est à ce titre que l'on peut parler d'une réglementation souple dans la mesure où elle laisse une importante marge de manœuvre aux acteurs de cette reconnaissance. Or on a pu voir que la difficulté d'appréciation de l'étendue du handicap, et du handicap mental en particulier, pouvait tout à fait permettre de rendre des décisions différentes d'une CDAPH à une autre tout en respectant ces conditions de forme. Cependant, il ne faut pas en conclure une absence de réglementation totale sur le fond même de la décision.

Il existe deux principaux outils d'harmonisation de nature réglementaire ayant vocation à être utilisés par les MDPH mais intervenant cependant à différents stades de la procédure de décision : le guide-barème, destiné à être utilisé par les CDAPH, et le guide d'évaluation des besoins de compensation, destiné, lui, à l'usage des équipes pluridisciplinaires, qui interviennent dans la phase d'instruction du dossier. Le guidebarème est un outil à destination des $\mathrm{CDAPH}$ qui a essentiellement vocation à donner une fourchette d'attribution d'un taux d'incapacité selon la gravité du handicap, appréciée sur des critères de conséquences sociales du handicap ${ }^{47}$. Si ce guide-barème permet de déterminer avec une certaine précision les incapacités les plus graves entraînant l'attribution de l'allocation adulte handicapé ${ }^{48}$, elle ne permet pas de déterminer l'existence ou non d'un handicap. En effet la reconnaissance administrative du handicap ne se limite pas à l'attribution de cette allocation, mais comprend également l'attribution de cartes d'invalidité, ou encore et surtout la reconnaissance de la qualité de travailleur handicapé qui constitue l'essentiel des décisions rendues par les MDPH. Ainsi ce guide ne permet pas avec précision de déterminer le champ du handicap. De plus, la dimension sociale que revêt la reconnaissance du handicap fait

47. Voir guide-barème en annexe 2-4 du CASF.

48. Qui constitue un minimum social pour les personnes dont le taux d'incapacité est reconnu comme étant au moins de $50 \%$. 
naître des difficultés spécifiques, notamment quant à l'interprétation de certaines dispositions législatives ou réglementaires. Ainsi, par exemple, concernant la reconnaissance de la qualité de travailleur handicapé, «avec l'évolution du marché du travail consécutive à la crise économique, il devient extrêmement difficile d'opérer la distinction entre le fait de ne pas pouvoir se procurer un emploi du fait du handicap, ce que voudrait la loi, et de ne pas pouvoir se procurer un emploi tout court ${ }^{49}$ ".

Comme le guide-barème, le Guide dévaluation des besoins de compensation de la personne handicapée (GEVA) a une portée réglementaire. C'est un décret du 6 février $2008^{50}$ qui en fait le support de l'évaluation des besoins de compensation de la personne handicapée. Contrairement au guide-barème, ce n'est donc pas un outil à destination des $\mathrm{CDAPH}$, mais des équipes pluridisciplinaires qui instruisent le dossier en amont de la décision. Ainsi, si les besoins de compensation pourront être librement estimés d'une MDPH à l'autre par les équipes pluridisciplinaires, ce guide permet au moins que ces besoins de compensation soient estimés sur la base des mêmes éléments. En revanche, le GEVA n'établit en aucun cas de fourchette d'appréciation du besoin de compensation mais se contente de lister les éléments qui doivent être pris en compte par l'équipe pluridisciplinaire selon la nature du handicap. Comme le souligne la CNSA, «le GEVA est un support majeur de la mise en place de l'égalité de traitement des demandes de compensation sur l'ensemble du territoire et de la culture commune autour du handicap ${ }^{51} »$.

Néanmoins, l'utilisation ou non de ces guides, qu'il s'agisse du guidebarème ou du GEVA, peut difficilement être imposée aux MDPH dans la mesure où leur non-utilisation ne peut permettre de remettre en cause la décision. Si leur utilisation est exigée règlementairement, leur présence formelle au dossier ne l'est pas. Cependant, le tribunal du contentieux de l'incapacité ${ }^{52}$ (TCI) qui est la juridiction compétente concernant la contestation du taux d'incapacité se fondera sur le dossier fourni à la MDPH pour estimer, à son tour, si la fixation du taux d'incapacité est justifiée au regard du guide-barème. Elle peut même aller jusqu'à se

49. Rapport d'information de l'Assemblée nationale $n^{\circ} 2542$, sur le fonctionnement des COTOREP, 12 juillet 2000.

50. Décret $\mathrm{n}^{\circ} 2008-110$ du 6 février 2008 relatif au guide d'évaluation des besoins de compensation des personnes handicapées.

51. Le GEVA, guide d'évaluation des besoins de compensation des personnes handicapées, Paris, Les Mémos de la CNSA, juin 2010.

52. La cour d'appel étant la Cour nationale de l'incapacité et de la tarification de l'assurance des accidents du travail (CNITAAT). 
fonder sur les seuls éléments fournis par le demandeur pour fixer ellemême le taux d'incapacité, en cas d'absence de transmission du dossier par la Maison départementale concernée ${ }^{53}$.

On peut donc estimer que le recours juridique constitue un important facteur d'harmonisation nationale dans le cadre de la reconnaissance du handicap mental. Ce serait sans doute vrai si le handicap mental ne constituait pas un obstacle majeur pour l'exercice d'un recours. En effet, on observe que les rares recours formés par des personnes handicapées mentales contre les décisions des $\mathrm{MDPH}$ le sont lorsque ces personnes sont placées sous tutelle familiale ${ }^{54}$ (parents, fratrie...) Ces recours apparaissent beaucoup plus rares lorsque le tuteur est désigné par un organisme privé de tutelle ou lorsque la personne handicapée mentale ne fait pas l'objet d'une mesure de protection. Or seulement $4 \%$ des personnes bénéficiant d'une reconnaissance administrative de leur handicap mental font l'objet d'une telle mesure de protection ${ }^{55}$.

Comme nous le voyons, les instruments réglementaires sont en euxmêmes insuffisants, étant donné la marge de manœuvre laissée ainsi que les difficultés d'accès aux recours juridiques, à garantir l'égalité de traitement sur l'ensemble du territoire, d'autant plus dans un contexte de diversification des acteurs aux intérêts potentiellement divergents au sein même des CDAPH mais également entre départements. Cependant, l'esprit de la loi de 2005 qui entend mettre l'usager au cœur de son orientation, rend nécessaire cette souplesse. Il y a donc là ce qui semble être un paradoxe entre une politique nationale qui réclame un traitement égal des usagers et la volonté de laisser une marge de manœuvre importante aux acteurs qui prendront, au final, la décision. Bien qu'ayant valeur réglementaire, ces deux guides sont donc davantage des éléments d'harmonisation des pratiques qu'une norme permettant d'assurer une unité dans le traitement des dossiers. Aussi, comme leurs noms l'indiquent, ces dispositions restent-elles des « guides » qui, s'ils constituent une référence nationale pour l'ensemble des $\mathrm{MDPH}$, ne peuvent, à eux seuls garantir une égalité de traitement des usagers. Il est donc apparu nécessaire de développer des moyens complémentaires pour garantir cette égalité, tout en conservant une certaine souplesse. Si certaines disparités risquaient d'être incompressibles en raison notamment de la spécificité des politiques sociales de chaque département, certaines

53. CNITAAT, section personnes handicapées, 14 février 2012.

54. Voir notamment CNITAAT, section personnes handicapées, 9 mars 2011.

55. Sylvie Renaut et Gilles Séraphin, «Les majeurs sous protection juridique: état des lieux », Recherches familiales, $\mathrm{n}^{\circ}$ 1, 2004. 
disparités pouvaient en revanche être grandement atténuées par la mise en réseau des acteurs chargés de la reconnaissance du handicap.

\subsection{La régulation par la coordination}

Si le terme de régulation est originellement apparu pour traiter des interventions de l'État dans le domaine économique, il apparaît que ces modes d'actions administratives ne sont plus réservés exclusivement à cette sphère ${ }^{56}$. En effet, l'instauration d'un dialogue et d'une prise de décision collective élaborée avec les acteurs principaux de la politique publique concernée semblent également ressortir de ce nouveau mode d'action publique. De même, l'instauration de contrôle a posteriori d'efficacité et d'effectivité de cette action sous forme de bilan vise à inciter les acteurs à adopter une conduite perçue comme la plus apte à garantir les objectifs fixés par les textes législatifs.

Les facteurs de décentralisation, d'autonomie des MDPH favorisent les disparités dans les décisions rendues par les départements. Nous venons de voir que les limites traditionnelles au pouvoir de décision des CDAPH sont relativement restreintes et que, si elles permettent l'émergence de références communes dans l'appréciation du handicap, elles ne sont pas à même de garantir l'égalité de traitement des usagers sur l'ensemble du territoire, dans la mesure où une certaine autonomie dans la prise de décision, en raison des facteurs précédemment évoqués apparaît irréductible. Pour Patrice Duran, «l'idée sous-jacente est que les institutions du gouvernement n'ont plus le monopole d'une action publique qui relève aujourd'hui d'une multiplicité d'acteurs dont la capacité d'action collective détermine la qualité, elle est prioritairement une interrogation sur le pilotage de l'action publique ${ }^{57}$ ». Ainsi la légitimité de l'action publique est aujourd'hui davantage fondée sur l'efficacité de cette action que sur sa légalité. Cela ne signifie pas que l'action administrative soit littéralement " hors la loi », mais que les caractéristiques de la loi et même des dispositions réglementaires en matière de handicap laissent volontairement une importante marge de manœuvre afin que les institutions chargées de la reconnaissance du handicap mettent en œuvre les mesures qui leur paraissent les plus efficaces pour atteindre l'objectif fixé. Cet objectif restant lui, en revanche, fixé par la loi. Aussi, une

56. Jacques Chevallier, «L'État régulateur», art. cité, p. 32.

57. Patrice Duran, "Action publique, action politique», dans Jean-Philippe Leresche (dir.), Gouvernance locale, coopération et légitimité, Paris, Pédone, p. 369-389, cité par Fabienne Leloup et al., «La gouvernance territoriale comme nouveau mode de coordination territoriale?», Géographie, économie, société, vol. 7, nº 4, 2005. 
nouvelle forme d'action a-t-elle été développée dans un but d'harmonisation des pratiques, cette fois non contraignante. Cette nouvelle forme d'action repose sur la coordination des différents acteurs entrant en jeu dans la politique d'insertion professionnelle des personnes handicapées, elle est « un pilotage de l'action publique». La coordination a d'ailleurs comme point de rapprochement notable avec l'expertise qu'elle repose sur une certaine maîtrise de l'information.

Les principaux supports de la coordination sont en effet l'établissement d'un réseau permettant de faire circuler l'information ainsi que la mise en place d'un organe chargé de la collecte et de l'analyse de l'ensemble des informations. Dans le cadre de la reconnaissance du handicap, cet organe jouant un rôle central se nomme la CNSA. La CNSA a été créée par la loi du 30 juin $2004^{58}$. La loi du 11 février 2005 viendra à la fois renforcer et préciser ces missions, notamment auprès des MDPH. Le rapport de l'Inspection générale des affaires sociales et de l'Inspection générale des finances de 2006 observe que « la situation actuelle évoque un certain dessaisissement de l'administration centrale de son rôle de pilotage au profit de la $\mathrm{CNSA}^{59}$ ». La Caisse nationale apparaît comme ayant désormais un rôle pivot concernant la coordination entre les différents acteurs, qui comme nous l'avons vu apparaissent de plus en plus diversifiés. Depuis cette loi, la CNSA apparaît comme ayant à la fois un rôle de gestion d'un fond d'aide aux personnes handicapées, notamment pour l'attribution de la prestation de compensation du handicap ( $\mathrm{PCH})$, mais également un important rôle de coordination du " réseau » des MDPH à travers une action d'évaluation de l'activité des $\mathrm{MDPH}$ ainsi que comme relais du pouvoir réglementaire. Nous avons vu précédemment qu'une infinité de personnes privées ou publiques pouvaient intervenir au sein des MDPH par le biais de conventions de partenariat établies avec celles-ci. Ces conventions de partenariat peuvent avoir certaines caractéristiques propres à assurer une coordination, tant sur le plan de l'effectivité des décisions de la CDAPH à travers son rôle d'instrument de suivi des décisions, que sur le plan de la mise en commun de moyens d'expertise. En effet l'ensemble des préconisations de la CDAPH ne s'imposent pas aux acteurs qui prennent la suite. Si par exemple, une orientation en établissement médico-social

58. Loi $\mathrm{n}^{0}$ 2004-626 du 30 juin 2004 relative à la solidarité pour l'autonomie des personnes âgées et des personnes handicapées.

59. Rapport IGF-IGAS n ${ }^{\circ}$ 2006-44 sur l'allocation adulte handicapé, 2006. 
s'impose à ces établissements ${ }^{60}$, à l'inverse, dans le cas d'une orientation vers le milieu ordinaire de travail ${ }^{61}$, les préconisations de la CDAPH ne jouent qu'un rôle de recommandations. Ainsi les prescriptions de la $\mathrm{CDAPH}$ en matière, par exemple, de secteur d'emploi ne s'imposent pas au service public de l'emploi. La CNSA remarque à cet égard que «c'est probablement dans le domaine de l'insertion professionnelle qu'il existe le plus grand nombre de conventions ${ }^{62} »$. Les acteurs qui interviennent sur le terrain de la formation des adultes handicapés regroupent à la fois des organismes spécifiques au handicap, comme l'Association de gestion du fonds pour l'insertion des personnes handicapées (AGEFIPH) et les dispositifs de formations de droit commun, comme l'association pour la formation professionnelle des adultes (AFPA). La question de l'insertion professionnelle et de la formation des adultes, handicapés ou non, fait également intervenir une nouvelle collectivité publique, la région, qui a, en la matière, une compétence d'attribution ${ }^{63}$. Ensuite, en termes quantitatifs, "les demandes liées à l'insertion professionnelle et à l'emploi représentent la moitié des demandes adressées aux MDPH s'agissant des adultes ${ }^{64} »$. Pour cette raison, de nombreux outils de suivi, à portée nationale comme à portée locale, sont développés par les différents acteurs de l'insertion professionnelle. C'est par exemple le dispositif « appui projet », financé par l'AGEFIPH et qui permet à une personne effectuant une première demande de reconnaissance de la qualité de travailleur handicapé de bénéficier d'une immersion en entreprise afin de l'aider à se déterminer dans ses choix d'orientation professionnelle. Ce dispositif permet également par la suite à la $\mathrm{CDAPH}$ de disposer pour sa prise de décision d'informations concrètes sur l'employabilité de la personne, de même que pour le service public de l'emploi qui peut être appelé à prendre le relais. Le suivi des décisions est également en partie effectif grâce aux partenariats entre les MDPH et les services médicosociaux. Bien que ces partenariats ne reposent pas sur une obligation légale mais sur une simple faculté, il apparaît que ce type de partenariats

60. C'est par exemple le cas pour les orientations en matière de scolarisation des enfants, qui s'imposent à l'enseignement public normal comme aux instituts médicoéducatifs (IME).

61. On parle de milieu ordinaire de travail par opposition au milieu protégé de travail, qui depuis la loi de 2005 ne comprend plus que les établissements et services d'aides par le travail (ESAT), encore connus sous l'appellation de centres d'aide par le travail (CAT).

62. CNSA, MDPH : 5 ans déjà !, op. cit.

63. Art. L 214-1 du Code de l'éducation.

64. Rapport CNSA précité. 
se développe souvent ${ }^{65}$ et permet le suivi des décisions. Ainsi est-il de plus en plus courant que les MDPH établissent des partenariats avec les établissements médico-sociaux permettant l'échange d'informations tant de listes d'attente à jour, que sur l'usager.

La diversité des partenariats en matière de dispositif d'insertion professionnelle des personnes en situation de handicap mental ne permet pas l'exhaustivité. Cependant ce mécanisme du développement des partenariats fait de la MDPH une sorte de carrefour d'échanges de données et permet un suivi effectif des décisions de la MDPH, les partenaires étant tenus par les contrats de partenariats de communiquer sur leurs actions. Cependant la contrepartie d'un tel fonctionnement qui laisse une place à une diversité d'acteurs peut être de rendre relativement trouble le rôle respectif de chacun des partenaires en matière d'insertion professionnelle pour l'usager.

De nombreux partenariats se développent également dans une perspective de mise en commun de moyens. Ainsi de nombreuses associations mettent à disposition leur expertise ainsi que des outils d'évaluation du handicap. Ces aides avec ou sans contrepartie financière sont très variables d'une MDPH à l'autre. Elles peuvent être une mise à disposition d'outils d'aide à l'orientation professionnelle comme un véritable diagnostic d'employabilité du demandeur. Par exemple, le projet de vie ${ }^{66}$ qui est un élément central de la participation de l'usager à la décision d'orientation le concernant instauré par la loi de 2005, reste facultatif. Concernant le handicap mental, l'élaboration du projet de vie peut poser problème, ne serait-ce que parce que les déficiences intellectuelles et profondes empêchent le plus souvent une acquisition des fondamentaux scolaires, dont la lecture et l'écriture. Cela se traduit par le fait que les projets de vie sont finalement rarement formalisés. La MDPH se voit confier la mission d'assurer une aide à la formulation du projet de vie ${ }^{67}$, sans pour autant qu'une autre disposition législative ou réglementaire prévoie que l'existence d'un tel organe soit mis en place au sein des MDPH. Ce projet de vie constitue cependant un des apports majeurs de la loi de 2005 relativement aux droits de l'usager. Pour pallier ce problème, des partenariats sont développés avec des associations chargées d'aider les usagers à la formalisation des projets de vie. Ainsi, par exemple,

65. CNSA, MDPH: 5 ans déjà !, op. cit.

66. Le projet de vie est un document écrit dans lequel le demandeur peut exprimer ses désirs d'orientation, mais également l'ensemble de ses souhaits, tels que les loisirs qu'il aimerait pratiquer.

67. Art. L 146-3 du CASF. 
l'association Inter Parcours propose-t-elle une aide à la formalisation des projets de vie. Cela pourrait se limiter à de simples initiatives locales. Cependant, en tant que "bonnes pratiques", la CNSA, à travers ses rapports, favorise ces initiatives en les relayant et les valorisant.

C'est ici qu'apparaît le rôle central de la CNSA en termes de coordination de l'action des MDPH. En effet, il existe une MDPH par département, soit 101. Il apparaît donc délicat d'établir une communication efficace entre les MDPH sans la création d'un organe chargé de collecter les données auprès des MDPH et de les analyser. L'une des caractéristiques des nouveaux registres de l'action publique est notamment l'établissement de bilans de l'effectivité et de l'efficacité d'une politique. "Ainsi l'évaluation de l'action publique ne se fait plus a priori dans le seul respect de principes de droit, mais a posteriori eu fonction de la manière selon laquelle la norme a réalisé les buts poursuivis par l'autorité publique. Dès lors, le droit devient évaluable sous un jour nouveau qui est celui de sa capacité à participer à la résolution des problèmes publics ${ }^{68}$.» Une des attributions majeures de la CNSA en matière d'harmonisation nationale des pratiques de l'ensemble des MDPH est celle «d'assurer un échange d'expériences et d'informations entre les maisons départementales des personnes handicapées [et] de diffuser les bonnes pratiques d'évaluation individuelle des besoins et de veiller à l'équité du traitement des demandes de compensation ${ }^{69}$ ». En d'autres termes, la CNSA est chargée, par le biais de rapports publics d'établir un diagnostic orienté de l'activité des différentes MDPH. Orienté, dans la mesure où l'objet même de ces rapports comparant les différentes pratiques est de « diffuser les bonnes pratiques", c'est-à-dire, sans pour autant établir un classement des différentes MDPH, d'identifier les «bons» et les «mauvais élèves». La diffusion de ces «bonnes pratiques» passent par des relais autrefois étrangers à l'action publique. Ainsi, la CNSA établit-elle des partenariats avec les médias, qu'il s’agisse de la presse généraliste ou spécialisée : elle a en 2011 répondu à plus de 170 sollicitations de la presse ${ }^{70}$.

La pratique de la mise en concurrence de différents établissements publics tend à se généraliser dans un contexte d'intégration des techniques entrepreneuriales à l'administration publique. Cependant, on ne peut pas estimer qu'il s'agisse véritablement d'une mise en concurrence dans le cas de la pratique des $\mathrm{MDPH}$, dans la mesure où le recours à l'une ou

68. Patrice Duran, "Piloter l'action publique, avec ou sans le droit? ", Politiques et management public, vol. 11, $\mathrm{n}^{\circ} 4,1993$.

69. Art. L 14-10-1 6 du CASF.

70. CNSA, rapport d'activité 2011. 
l'autre MDPH ne relève pas d'un choix de la part de l'usager mais de son lieu de résidence ${ }^{71}$ et que d'autre part les rapports sur le fonctionnement de ces maisons départementales ne les désignent pas nominativement. Ils ont plutôt une fonction incitative pour les MDPH qui auraient un fonctionnement qui s'écarte des «bonnes pratiques » valorisées dans ces rapports. Par exemple, parmi les bonnes pratiques, la formalisation du plan personnalisé de compensation ou la rencontre des demandeurs par les équipes pluridisciplinaires permettent en même temps que de mettre en lumière les disparités existantes, d'inciter les MDPH qui s'écartent le plus de ces «bonnes pratiques", à améliorer leur fonctionnement. Par la valorisation de pratiques favorables aux usagers dans le traitement de leurs demandes, un tel système a comme principal avantage qu'il permet un nivellement «par le haut» des pratiques des MDPH. Ce n'est pas en effet le seul respect de l'esprit de la loi de 2005 qui est valorisé à travers ces rapports, mais plus encore un « esprit d'initiative » des MDPH dans une démarche qualitative. Tout en étant conformes à l'esprit de la loi de 2005 qui entend favoriser l'écoute de l'usager et sa participation à sa propre orientation, ces «bonnes pratiques » valorisées par les CNSA n'ont pas en revanche de fondement juridique. Ces «bonnes pratiques » reposent davantage sur l'appréciation de critères d'efficacité de l'activité des MDPH sur un plan qualitatif et quantitatif (aide aux usagers, développement de partenariats, délai de traitement des dossiers...)

Enfin, la CNSA, dans ses relations avec les MDPH joue un rôle d'appui quant à l'utilisation des différents guides réglementaires et est chargée de leur élaboration formelle. Nous avons vu précédemment que l'efficacité des différents guides mis en place par le pouvoir réglementaire dans un but d'harmonisation des pratiques se trouvait limitée par la marge de manœuvre que laissent nécessairement ces outils aux MDPH, qu'il s'agisse des équipes pluridisciplinaires ou de la CDAPH. Une des limites principales de ces outils tient à l'interprétation qui en est faite, dans la mesure où la volonté d'individualisation de l'action administrative rend difficilement uniforme leur interprétation, et où la technicité de ces documents nécessite une appropriation de l'outil par les équipes pluridisciplinaires et les CDAPH. C'est à ce titre que la CNSA intervient. En effet, au terme de l'article L 14-10-1 $3^{\circ}$ du CASF, une des missions de la CNSA est «d'assurer un rôle d'expertise technique et de proposition pour les référentiels nationaux d'évaluation des déficiences et de la perte d'autonomie, ainsi que pour les méthodes et outils utilisés pour apprécier

71. Art. L 146-10 du CASF. 
les besoins individuels de compensation ». La CNSA joue à ce titre le rôle de relais du pouvoir réglementaire. C'est notamment elle qui diffuse ces guides sous la forme de documents téléchargeables et prêts à l'emploi par le biais de son site. De même, c'est elle qui se fait l'écho de l'utilisation concrète du GEVA par les maisons départementales et des critiques et améliorations qui pourraient y être apportées. Elle est donc un relais du pouvoir réglementaire, mais elle fait également remonter au pouvoir réglementaire les informations sur les limitations que les $\mathrm{MDPH}$ ellesmêmes pourraient rencontrer dans l'application de ces règlements.

En conséquence, la procédure de reconnaissance du handicap mental de l'administration apparait comme de moins en moins dépendante du pouvoir central, mais d'acteurs de plus en plus nombreux. Si cette diversification des acteurs peut permettre une meilleure prise en compte de la situation spécifique de l'usager, cela appelle cependant une remarque quant au risque de disparités entre départements, d'autant qu'en raison notamment du caractère conciliatoire et du rôle de l'expertise dans la prise de décision des CDAPH, l'inefficacité des limites classiques au pouvoir de l'administration (réglementation et contrôle du juge) apparaissent comme un obstacle majeur à une unité territoriale. À défaut d'unité, c'est donc une harmonisation qui est recherchée à travers des moyens plus souples que les moyens classiques, qui passent notamment par un " pilotage » des MDPH. Une égalité de traitement stricte apparaît alors impossible en raison des différences même de populations des départements et du principe de libre administration des collectivités territoriales. Cela ne doit pas pour autant faire oublier que l'ancien système des COTOREP était loin de donner des résultats satisfaisants ${ }^{72}$, bien que l'administration centrale y fût alors prédominante. Néanmoins la prise en compte de l'ensemble des acteurs dans une structure qui englobe l'ensemble des aspects de la situation des personnes handicapées pose un problème de lisibilité de la politique du handicap, aggravé par des textes relativement disparates, le handicap étant susceptible d'avoir des répercussions sur l'ensemble de la vie sociale et donc dans de nombreuses branches du droit ${ }^{73}$. Si nous avons pu voir le rôle pilote de la CNSA concernant la coordination des MDPH, la CNSA n'a pas ce rôle pour l'ensemble des aspects de la politique du handicap. Par exemple, pour les établissements médicalisés, ce sont désormais les ARS

72. Rapport [...] sur le fonctionnement des COTOREP, op. cit.

73. Pour le seul aspect de l'insertion professionnelle des personnes handicapées, il est ainsi nécessaire de se référer au minimum au Code de l'éducation, au CASF, au Code du travail ainsi qu’au Code de la sécurité sociale. 
qui assurent un rôle de pilotage ${ }^{74}$. La politique du handicap se caractérise par un entrelacement des compétences entre l'État, les différentes collectivités publiques et les autorités administratives indépendantes. L'individualisation de la reconnaissance du handicap par les commissions décisionnaires (les $\mathrm{CDAPH}$ ) des maisons départementales nécessite en effet le développement de nouveaux moyens d'harmonisation des décisions que ces instances peuvent prendre. La régulation apparaît alors comme le meilleur moyen de garantir la cohérence de la reconnaissance du handicap mental sur l'ensemble du territoire dans l'esprit de la loi de $2005^{75}$.

Doctorante en droit public, université de Rouen

Centre d'études des systèmes juridiques

74. Loi $\mathrm{n}^{0} 2009-879$ du 21 juillet 2009 portant réforme de l'hôpital et relative aux patients, à la santé et aux territoires.

75. Je remercie Carole Nivard, maître de conférences à l'université de Rouen ainsi que Jean-Philippe Bras, professeur à l'université de Rouen, pour leurs conseils précieux lors de la réalisation de cet article. 